\title{
Long-term Results of in Situ Pinning Treatment of Femoral Head Slippage Patients
}

\author{
Kayahan Karaytuğ' ${ }^{1}$, Gökhan Polat², Turgut Akgül'², Ali Asma³, Cengiz Şen², Mehmet Aşık²
}

\begin{abstract}
Introduction: Slipped capital femoral epiphysis (SCFE) is a disease that occurs as a result of the posterior and inferior displacement of the femur head. SCFE is the most common hip pathology in adolescence. It is classified as stable and unstable SCFE according to the loadability of the affected leg. The widely accepted method for stable SCFE treatment is in situ pinning. In situ pinning is an effective method for relieving pain in the early period and preventing the progression of slippage. On the other hand, because of the non-anatomic reduction of physis, it is expected in middle and long-term osteoarthritis. Our aim in this study is to report the long-term functional outcomes of patients with SCFE who were treated with in situ fixation at a trauma center.
\end{abstract}

Methods: This study was designed as a retrospective collection of data at a tertiary trauma center, and descriptive research based on prospective assessment and observation. Patients who were treated with closed-loop in situ pinning were evaluated retrospectively for acute, subacute, and chronic slip intensity according to the Southwick classification as mild, moderate, severe, and load-failing. Patient satisfaction surveys (SF-12, WOMAC, HHS, and HOS) were used to evaluate postoperative clinical and functional outcomes.

Results: Overall, 33 patients were included in the study, and 38 hips (28 unilateral, 5 bilateral) were treated surgically. In total, 11 patients (33.3\%) had no complaint. In 11 patients (33.3\%), the main complaint was limping. The main complaint of 7 patients (21.2\%) was restricted movement, and 4 patients (12.1\%) were found to have underlying pain. The mean functional results of the patients after the first surgical treatment were HHS, 82.6; HOS, 87.2; WOMAC, 82.8; SF-12 PCS, 43.8. The SF-12 MCS was found to be 48.2.

Conclusion: In this study, satisfactory results were obtained early in the in situ pinning method, and it was not enough to prevent osteoarthritis but other morbid complications in the long term.

Keywords: SCFE, adolescence, in situ pinning, osteoarthritis, secondary surgery

This study was presented in $25^{\text {th }}$ National Turkish Orthopedics and Traumatology Congress, 27 October-1 November 2015, Antalya, Turkey.

${ }^{1}$ Clinic of Orthopedics and Traumatology, Sarıkamıș State Hospital, Kars, Turkey ${ }^{2}$ Department of Orthopedics and Traumatology, İstanbul University İstanbul School of Medicine, İstanbul, Turkey

${ }^{3}$ Department of Orthopedics and Traumatology, Dokuz Eylül University School of Medicine, İzmir Turkey

Address for Correspondence:

Kayahan Karaytuğ

E-mail: kayhan_karaytug@hotmail.com

Received: 22.04.2017

Accepted: 15.11.2017

C Copyright 2018 by Available online at istanbulmedicaljournal.org

\section{Introduction}

The most common hip problem in adolescence is slipped capital femoral epiphysis (SCFE). SCFE is generally more prevalent in males aged 10-16 years $(1,2)$. Although its etiology has not been fully clarified, obesity, male gender, and endocrine anomalies are accepted as risk factors (3).

In the anatomy of SCFE, the slipped femoral head is displaced to the posterior and inferior of the femur neck. Varus, external rotation, and extensional deformities are observed in the proximal part of the femur because of this placement of the femur head (4).

Although many systems are used in the classification of SCFE today, the most preferred classification system is based on the stability of the physis. According to this classification, stable SCFE is said to be present if weight can be put on the injured extremity, and unstable SCFE is present if it cannot (5).

While in-situ fixation method is widely applied in the treatment of stable SCFE, gentle reduction and internal fixation (6) are performed in the treatment of unstable SCFE. Slipping may be prevented after the treatment, but the femur head may be found in a different localization from the point that it should be in anatomically. This malposition of the physis can lead to a clinically insignificant loss of internal rotation over time along with the patient's remodeling activity, or lead to impingement of the femur head in the acetabulum and to the development of premature osteoarthritis $(4,7)$.

To prevent postoperative femoral head malposition and hip dysfunction, some authors have proposed primary osteotomy $(8,9)$. Some authors have also suggested open reduction and internal fixation, but these reports mostly comprise short-term results (10-12).

Our purpose herein is to report the long-term functional outcomes of patients with SCFE who have been treated with in-situ pinning at our clinic. At the end of a 7-year follow-up period, in-situ pinning was observed to be effective in decreasing the complaints of patients and was a successful method in terms of early-period results. In medium- to long-term follow-ups, it has been concluded that it is a method with unfavorable functional results and in which secondary surgical treatment is needed. 


\section{Methods}

This study has been designed as a descriptive research based on gathering of retrospective information and prospective evaluation and observation at a tertiary center. Between January 1996 and December 2009, patients who were operated with in-situ pinning with a diagnosis of SCFE were included in this study. The approvals of all patients included in the study and their relatives have been obtained with Informed Consent Forms. This study was conducted in accordence with the Declaration of Helsinki. For in-situ pinning, 1, 2, or 3 half-threaded cannulated screws were used. To determine the acute, subacute, and chronic slip severity in patients at the time of admission, patient files were retrospectively analyzed in terms of being stable/unstable with the Southwick classification (mild $<33 \%$, moderate $33 \%-50 \%$, severe $>50 \%$ ). Postoperative follow-up information of the patients, functional status, need for secondary surgery, and complications were retrospectively questioned. The follow-up durations of the patients who underwent secondary surgery were ended on the date of the second operation and evaluated with the information obtained after the first treatment. Patient satisfaction surveys (SF-12, WOMAC, HHS, and HOS) were used to evaluate postoperative clinical and functional outcomes.

\section{Results}

Thirty-three patients were included in the study, of whom surgery was performed on 38 hips (28 unilateral, 5 bilateral). The mean age at the time of admission was 12.3 (range, 10-14) years. The mean follow-up time was calculated as 83.9 months (range, 18-216). At the time of admission, 9 (23.7\%) hips were evaluated as acute, 14 (36.8\%) as subacute, and 15 (39.4\%) as chronic SCFE. According to the clinical complaints of the patients, the slip was evaluated as mild in 24 (63.1\%) hips, moderate in 11 (28.9\%), and advanced in 3 (7.9\%). In-situ pinning was performed in 28 (73.6\%) hips with a single-cannulated screw (Figure 1), in 9 (23.7\%) hips with 2 cannulated screws, and in $1(2.7 \%)$ hip with 3 cannulated screws. When the main complaints were evaluated in the last control and before the secondary surgery, 11 (33.3\%) patients had no complaints. The main complaint was found to be limping in 11 (33.3\%) patients. The main complaint in 7 (21.2\%) patients was limitation of movement ability, and the complaint in $4(12.1 \%)$ patients was pain

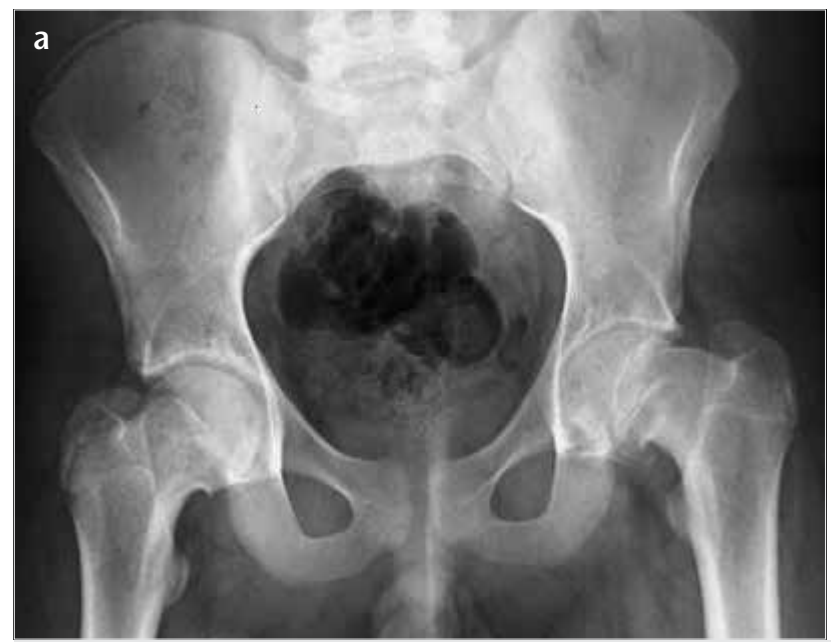

(Graph 1). In the follow-up period, secondary surgery was offered to $11(33 \%)$ patients with severe pain and limitation of movement, of whom 8 patients accepted the surgery. Three of the 8 patients were treated with arthroscopic femoroplasty (Figure 2), 2 patients with subtrochanteric valgisation-extension osteotomy, 1 patient with intraarticular osteotomy, and 2 patients with bilateral hip safe dislocation and femoroplasty. According to the patient-reported outcome measure, the mean values of the functional outcomes of the patients after the first surgical treatment were as follows: HHS 82.6, HOS 87.2, WOMAC 82.8, SF-12 PCS 43.8, and SF-12 MCS 48.2.

\section{Discussion}

Slipped capital femoral epiphysis is one of the most commonly encountered adolescent hip problems, and it is commonly seen in males within the age range of $10-16$ years $(1,2)$. Although its etiology is unclear, obesity, endocrine anomalies, and male gender have been found as the most significant risk factors (3). The incidence of SCFE, which shows parallelism with increased obesity in the USA, denotes that obesity is a risk factor (13).

When the pathoanatomy of the disease is examined, it is observed that the femoral head epiphysis is displaced posterior and inferior to the femur metaphysis. This causes extension, varus, and external rotation deformities in the proximal femur (4).

Considering the risk of avascular necrosis, the commonly accepted treatment modality for SCFE is scope-assisted gentle reduction and in-situ pinning of the physis (6). Publications related to SCFE in-situ pinning, with long follow-up durations, have revealed the results of older treatment methods (follow-up, casting, primary osteotomy, closed reduction, and pinning) $(3,4,6)$.

In current practice, in-situ pinning prevents further slipping of SCFE, but it can cause significant problems in the hip joint due to the non-anatomic position of the epiphysis. With the help of remodeling activity (4), the results of this non-anatomical position may not be clinically meaningful; however, the femoral head sometimes poses a risk for the development of premature osteoarthritis by causing impingement in the acetabulum because of inadequate remodeling capacity (7). For this reason, osteotomies

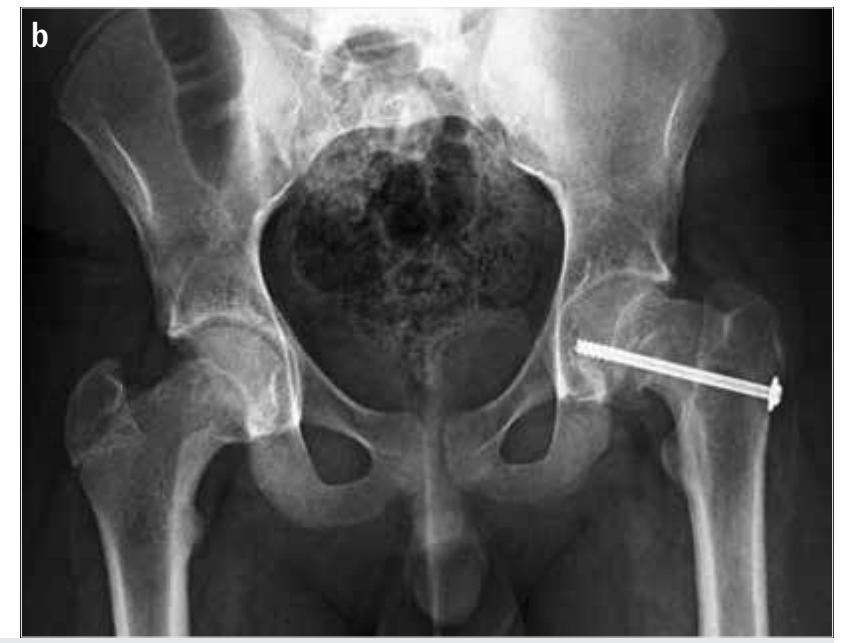

Figure 1. a, b. Preoperative (a) and postoperative (b) graphs of a 11-year-old male patient in whom in-situ pinning was performed with a singlecannulated screw 

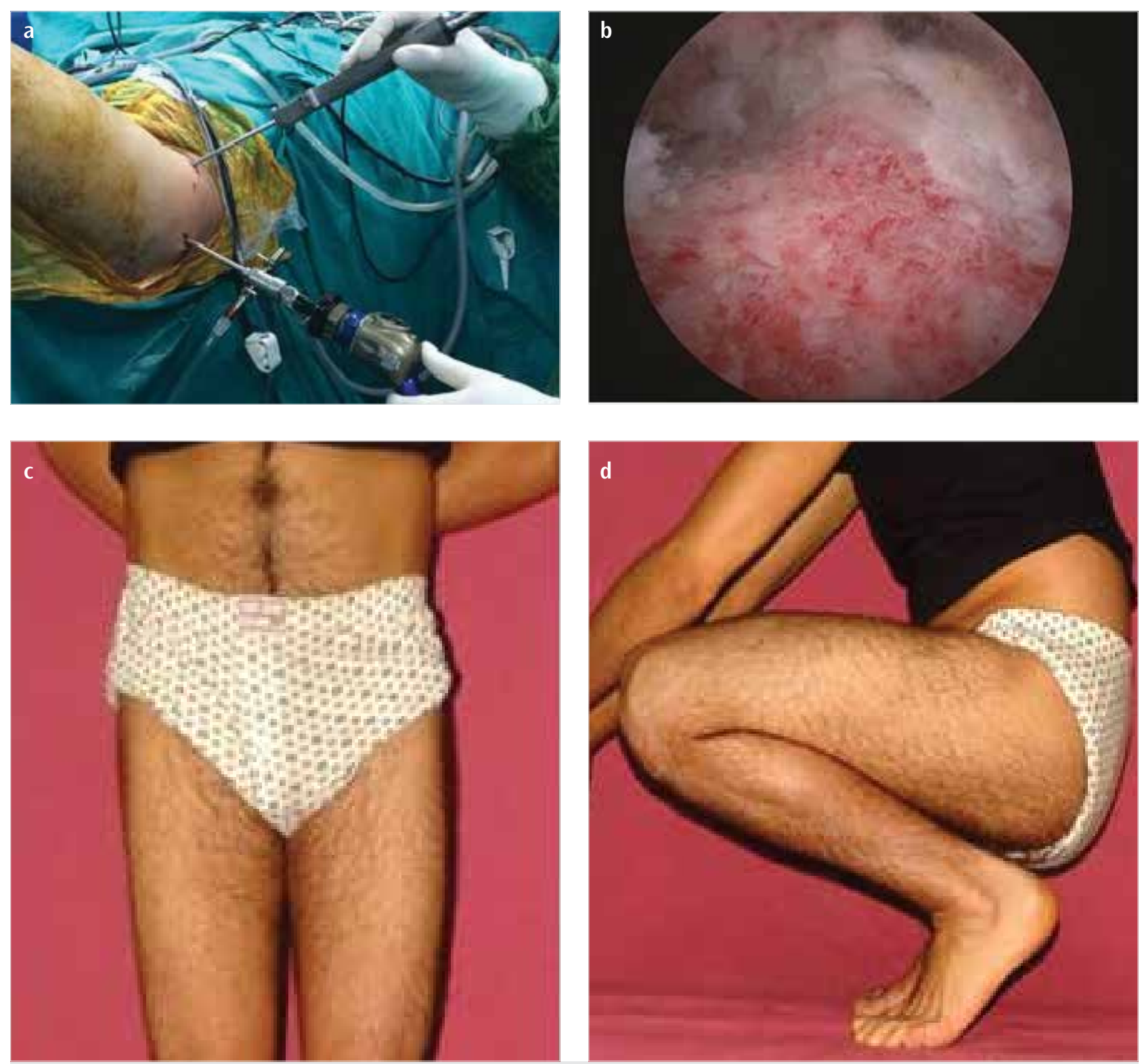

Figure 2. a-d. Intraoperative (a, b) and postoperative 1st year clinical images (c, d) of a patient who underwent arthroscopic femoroplasty due to the limitation of movement ability

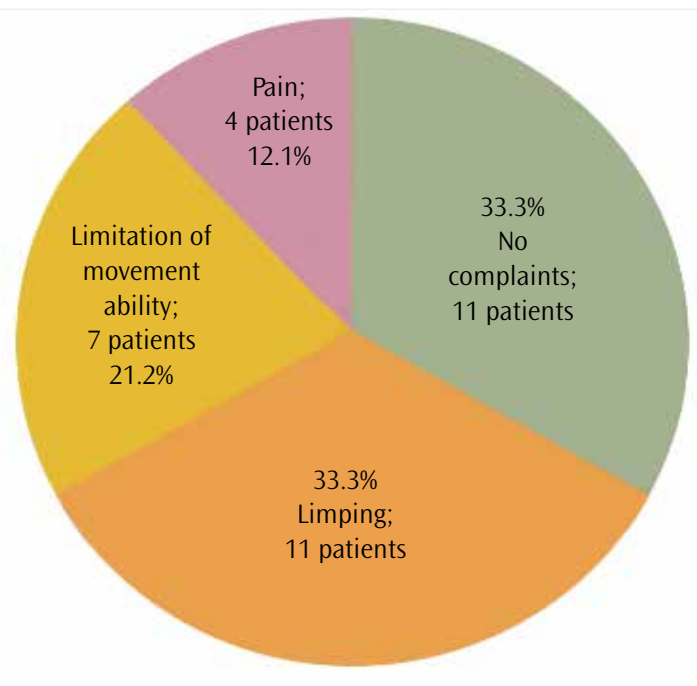

have been described as a primary treatment modality to prevent the development of femur deformity after SCFE and to prevent the restriction of hip function $(8,9)$. Recently, the short-term results of open reduction and internal fixation, suggested as the first treatment for stable and unstable SCFE, have been reported by some authors (10). Goodman et al. (14) revealed a strong association between post-slip morphology (pistol grip and femoral head tilt deformity), depending on post-SCFE remodeling failure, and early osteoarthritis development. Anterior acetabulum flattening, which corresponds to antero-superior femoroacetabular impingement (FAI) morphology, describes cystic degeneration and global osteoarthritis in the anterior epiphyseal-metaphyseal region (14, 15). Furthermore, early osteoarthritic changes, cartilage and labral pathologies may occur not only in the moderate-severe stage but also in mild SCFE (16). There are limited publications associated with in-situ pinning with long follow-up periods. However, some of these studies have reported excellent results regarding pinning in mild SCFE cases (17). 
In contrast, many studies have shown that the early development of osteoarthritis is inevitable after SCFE, regardless of slip severity $(18,19)$.

In their retrospective study, Castaneda et al. (20) reported radiological osteoarthritis development in all patients independently of the slip severity at the end of an average follow-up duration of 22.3 years. In a long-term functional and radiological study, Larson et al. (12) indicated that although worse clinical outcomes were obtained in patients with severe slips, patients with mild SCFE also became symptomatic over time.

In the results of a retrospective, multicenter study conducted by Poorter et al. (21), it was indicated that short- and long-term outcomes of in-situ pinning after a mild-moderate slip are good; however, clinical outcomes are poor after severe slip. Thus, open reduction internal fixation should be considered for advanced slips. After a mean follow-up duration of 18 years, they concluded that good results are obtained with in-situ pinning in patients with a mild-moderate slip; thus, no open procedures were necessary. Another important implication that Larson et al. (12). pointed out is that secondary reconstructive surgical intervention is needed at high rates, and pain persists during mild-moderate slips. While one-third of the patients had complaints about residual pain, secondary reconstructive surgical intervention was needed in $10 \%$ of the patients during the first 10 years of follow-up. At the end of 20 years, the rate of patients who underwent total hip arthroplasty due to osteoarthritis was $5 \%$ (12). In our patient group, the rate of necessity for secondary reconstructive surgery was found to be $33 \%$, and total hip arthroplasty was performed in none of these patients. Methods such as arthroscopic osteochondroplasty, safe hip dislocation (SHD), and intertrochanteric osteotomies were performed as secondary reconstructive surgeries in patients with FAI findings and symptoms after recovered SCFE (22). In the literature, arthroscopic femoral neck osteochondroplasty is recommended for mild slips with an angle smaller than $15^{\circ}$, and osteochondroplasty may be performed with a limited anterior approach (23) in cases where the metaphyseal hump cannot be removed completely and where the angle of slip is between $15^{\circ}$ and $30^{\circ}$. SHD provides greater access to the metaphyseal hump than arthroscopy and limited anterior approach in advanced slip degrees. In addition, it is easy to reveal labral and acetabular cartilage pathologies with SHD. Sink et al. (18) pointed out that labral and acetabular cartilage damage can be seen at very high rates after SCFE. Therefore, it has been stated that SHD is more appropriate for patients in whom a $15^{\circ}-30^{\circ}$ slip is detected and who have acetabular cartilage and labrum pathology and that they should be performed by experienced teams, considering the risk of avascular necrosis (18).

In-situ pinning after SCFE has been used extensively in the acute phase in order to prevent the complaints of pain and to prevent the increase in the degree of slip. Osteoarthritis development has radiologically been shown also in patients with mild slip in the long term after pinning (12). Although its effect in the clinic is not significant in patients with mild-moderate slip in the short term, clinical and radiologic osteoarthritis development is expected in patients with severe slip. According to long-term follow-up results, residual pain (12\%-33\%), limping, and the necessity of new surgical reconstructions (10\%-33\%) within 10 years constitute the negative aspects of this treatment method in patients in whom in-situ pinning is performed.
Ethics Committee Approval: Authors declared that the research was conducted according to the principles of the World Medical Association Declaration of Helsinki "Ethical Principles for Medical Research Involving Human Subjects", (amended in October 2013).

Informed Consent: Written informed consent was obtained from patients who participated in this study.

Peer-review: Externally peer-reviewed.

Author contributions: Concept - K.K., G.P., A.A.; Design - K.K., G.P., A.A.; Supervision - G.P., T.A., M.A; Resource - G.P., K.K., A.A.; Materials - K.K., C.S, M.A.; Data Collection and/or Processing - K.K., T.A, A.A.; Analysis and/or Interpretation - K.K., G.P., T.A.; Literature Search - C.Ş., K.K., M.A.; Writing K.K., G.P., T.A.; Critical Reviews - K.K., G.P., T.A.

Conflict of Interest: The authors have no conflict of interest to declare.

Financial Disclosure: The author declared that this study has received no financial support.

\section{References}

1. Bhatia NN, Pirpiris M, Otsuka NY Body mass index in paitents with Slipped capital femoral epiphysis. J Pediatr Orthop 2006; 26: 197-9. [CrossRef]

2. Lehmann CL, Arons RR, Loder RT, Vitale MG. The epidemiology of slipped capital femoral epiphysis: an update. J Pediatr Orthop 2006; 26: 286-90. [CrossRef]

3. Jingushi S, Suenega E. Slipped capital femoral epiphysis: etiology and treatment. J Orthop Sci 2004; 9: 214-9. [CrossRef]

4. Aronsson DD, Loder RT, Breur GJ, Weinsteinn SL. Slipped capital femoral epiphysis: current concepts. J Am Acad Orthop Surg 2006; 14: 66679. [CrossRef]

5. Loder RT, Richards BS, Shapiro PS, Reznick LR, Aronsson DD. Acute slipped capital femoral epiphysis: The importance of physeal stability. J Bone Joint Surg Am 1993; 75: 1134-40. [CrossRef]

6. Loder RT, Dietz FR. What is the best evidence for the treatment of slipped capital femoral epiphysis? J Pediatr Orthop 2012; 32: 158-65. [CrossRef]

7. Leunig M, Casillas MM, Hamlet M, Hersche O, Nötzli H, Slongo T. Slipped capital femoral epiphysis:early mechanical damage to the acetabular cartilage by a prominent femoral metaphysis. Acta Orthop Scand 2000; 71: 370-5. [CrossRef]

8. Southwick WO (1967) Osteotomy through the lesser trochanter for slipped capital femoral epiphysis.J Bone Joint Surg Am 1967; 49: 80735. [CrossRef]

9. Witbreuk MM, Bolkenbaas M, Mullender MG, Sierevelt IN, Besselaar PP. The results of downgrading moderate and severe slipped capital femoral epiphysis by an early Imhauser femur osteotomy. J Child Orthop 2009; 3: 405-10. [CrossRef]

10. Alves C, Steele M, Narayanan U, Howard A, Alman B, Wright JG. Open reduction and internal fixation of unstable slipped capital femoral epiphysis by means of surgical dislocation does not decrease the rate of avascular necrosis: a preliminary study.J Child Orthop 2012; 6: 277 83. [CrossRef]

11. Tibor LM, Sink EL. Risks and benefits of the modified Dunn approach for treatment of moderate or severe slipped capital femoral epiphysis.J Pediatr Orthop 2013; 33: 99-102. [CrossRef]

12. Larson AN, Sierra RJ, Yu EM, Trousdale RT, Stans AA. Outcomes of slipped capital femoral epiphysis treated with in situ pinning. J Pediatr Orthop 2012; 32: 125-30. [CrossRef]

13. Murray AW, Wilson NI. Changing incidence of slipped capital femoral epiphysis: A relationship with obesity? J Bone Joint Surg Br 2008; 90: 92-4. [CrossRef] 
14. Goodman DA, Feighan JE, Smith AD, Latimer B, Buly RL, Cooperman DR. Subclinical slipped capital femoral epiphysis. Relations to osteoarthrosis of the hip. J Bone Joint Surg Am 1997; 79: 1489-97. [CrossRef]

15. Fraitzl CR, Kafer W, Nelitz M, Reichel H. Radiological evidence of femoracetabular impingement in mild slipped capital femoral epiphysis: a mean follow-up of 14.4 years after pinning in situ. J Bone Joint Surg Br 2007; 89: 1592-6. [CrossRef]

16. Tscholl PM, Zingg PO, Dora C, Frey E, Dierauer S, Ramseier LE. Arthroscopic osteochondroplasty in patients with mild slipped capital femoral epiphysis after in situ fixation. J Child Orthop 2016; 10: 25-30. [CrossRef]

17. Boero S,Brunenghi GM,Carbone M, Stella G, Calevo MG. Pinning in slipped capital femoral epiphysis:long-term follow-up study.J Pediatr Orthop B 2003;12: 372-9. [CrossRef]

18. Sink EL, Zaltz I, Heare T, Dayton M. Acetabular cartilage and labral damage observed during surgical hip dislocation for stable slipped capital femoral epiphysis. J Pediatr Orthop 2010; 30: 26-30. [CrossRef]

19. Dodds MK, McCormack D, Mulhall KJ. Femoracetabular impigement after slipped capital femoral epiphysis:does slip severity predict clinical symptoms? J Pediatr Orthop 2009; 29: 535-9. [CrossRef]
20. Castañeda P1, Ponce C, Villareal G, Vidal C. The natural history of osteoarthritis after a slipped capital femoral epiphysis/the pistol grip deformity. J Pediatr Orthop 2013; 33: 76-82. [CrossRef]

21. de Poorter JJ, Beunder TJ,Gareb B, Oostenbroek HJ, Bessems GH, van der Lugt JC, et al. Long-term outcomes of slipped capital femoral epiphysis treated with in situ pinning. J Child Orthop 2016; 10: 371-9. [CrossRef]

22. Kuzyk PR, Kim YJ, Millis MB. Surgical Management of Healed Slipped Capital Femoral Epiphysis. J Am Acad Orthop Surg 2011; 19: 667-77. [CrossRef]

23. Sekiya JK, Wojtys EM, Loder RT, Hensinger RN. Hip artrhoscopy using a limited anterior exposure: An alternativeapproach for arthroscopic access. Arthroscopy 2000; 16: 16-20. [CrossRef]

Cite this article as: Karaytuğ K, Polat G, Akgül T, Asma A, Şen C, Aşık M. Long-term Results of In Situ Pinning Treatment of Femoral Head Slippage Patients. İstanbul Med J 2018; 19: 124-8. 